\title{
Density-functional calculations on singly and doubly excited Rydberg states of many-electron atoms
}

\author{
Amlan K. Roy and Shih-I Chu \\ Department of Chemistry, University of Kansas and Kansas Center for Advanced Scientific Computing, Lawrence, Kansas 66045
}

(Received 1 November 2001; revised manuscript received 11 February 2002; published 7 May 2002)

\begin{abstract}
Nearly 100 low-, moderately high-, and high-lying singly and doubly excited states of $\mathrm{He}$, Li, and Be have been calculated using a nonvariational, work-function-based exchange potential within the nonrelativistic Hohenberg-Kohn-Sham density-functional theory (DFT). The nonlinear gradient included in the Lee-Yang-Parr correlation functional is used to incorporate the correlation potential. The generalized pseudospectral method is used for nonuniform and optimal spatial grid discretization and solution of the Kohn-Sham equation to obtain accurate eigenvalues, expectation values, and radial densities for both ground and excited states. The results are compared with the available theoretical and experimental data. The discrepancy in the calculated singly excited state energies is within about $0.01 \%$ for He (for other atoms, it is less than $0.2 \%$ ), while that for the doubly excited states of $\mathrm{He}$ is well within $3.6 \%$. The deviations in the calculated single- and doubleexcitation energies for 31 selected states are in the error ranges $0.009-0.632 \%$ and $0.085-1.600 \%$, respectively. The overall agreement of the present results is quite gratifying, especially in the light of DFT's difficulties in dealing with excited states. The exchange-only results are practically of Hartree-Fock quality, and the correlation-included results are usually slightly overestimated. The present method offers a simple, computationally efficient and general scheme for accurate calculation of multiply excited Rydberg states within DFT.
\end{abstract}

DOI: 10.1103/PhysRevA.65.052508

PACS number(s): 31.15.Ew, 32.80.Rm, 32.80.Wr

\section{INTRODUCTION}

In the past decades density-functional theory (DFT) [1,2] has found wide applications in many areas in theoretical physics and chemistry, especially in the electronic structure calculations of atoms, molecules, and solids $[3,4]$. However, the majority of the applications are restricted to the ground states. There are several problems associated with the calculation of excited states within DFT, viz., the complete abandoning of the state-function concept, the unavailability of the Hohenberg-Kohn theorem for a general excited state parallel to the ground state, and the lack of accurate exchangecorrelation (XC) energy density functionals, etc. Moreover, while the highest-occupied orbital energy corresponds to the negative of the ionization potential (for well-behaved potentials vanishing at infinity), energies of other occupied and unoccupied orbitals have no rigorous correspondence to the excitation energies. Nevertheless, it has been shown recently [5] that the unoccupied true Kohn-Sham eigenvalues can also provide good excitation energies (which the commonly used approximate density functionals usually do not satisfy), of course, not to mention the cumbersome wave function and Hamiltonian orthogonality requirements met in any quantum mechanical calculations of excited states. Nevertheless, numerous attempts ([3]; see [6] for a review) have been made in this direction over the years; e.g., the ensemble densityfunctional method [7-11], the method based on partitioning of the wave function [12], the time-dependent (TD) DFT approach [13-16], etc. Although good results have been reported for singly excited states of low-lying states, to our knowledge, few results exist for multiply excited states, e.g., the doubly, and triply excited states, particularly those involving Rydberg excitation of many-electron systems, using these methods.
Recently, an exchange potential based on the workfunction approach $[17,18]$ was proposed for excited state calculation in many-electron atomic systems. The method is simple, computationally efficient, and has shown considerable success for some singly, doubly, and triply excited states, low and moderately high states, and valence and core excitations, as well as the autoionizing and satellite states ([19-23]; see [6] for a review). The work-function formalism is not derived from the usual variational principle for energy in the sense that the exchange potential is not expressible as $\delta E_{x}[\rho] / \delta \rho$ [as in the usual Kohn-Sham (KS) DFT]; rather, it is based on the physical interpretation that the electron moves in the electrostatic potential arising from the Fermi hole charge distribution. Further, it has been shown [24] that the spin-polarized version of the workfunction exchange potential can be obtained as an approximation to the accurate spin-polarized X-only KS potential, viz., the approximate optimized effective potential (OEP). This approximate OEP is an accurate representation of the exact numerically derived X-only OEP, obtained analytically based on the Green's function analysis of the OEP integral equation. It satisfies many key analytic features of the exact KS potential, viz., it reduces to the exact KS result in the inhomogeneous electron-gas limit, approaches $-1 / r$ as $r$ goes to infinity, obeys Koopman's theorem, etc.

All the previous DFT calculations [6,19-23] using the work-function approach, however, have employed a Numerov-type finite-difference (FD) scheme for the discretization of the spatial coordinates and the solution of the Kohn-Sham equation. Due to the existence of the Coulomb singularity at the origin and the long range nature of the Coulomb potential, the FD method requires a large number of grid points to achieve reasonable accuracy even for ground state calculations. In this article, we employ a different numerical method, namely, the generalized pseudospec- 
tral (GPS) procedure [25-27], to achieve high accuracy as well as to facilitate the Rydberg state calculations. The GPS method allows nonuniform spatial grid discretization and considerably more accurate calculations of eigenvalues and wave functions using only a modest number of grid points. The GPS method and its time-dependent extension [28] have been applied successfully to the study of electronic structure [29], and multiphoton dynamics of many-electron atoms and molecules in intense laser fields [30,31], as well as to the study of high resolution spectroscopy of Rydberg $\mathrm{H}$ atoms in external fields [32]. The main motivation of this article is to explore the feasibility of extending the GPS method and the work-function formalism for accurate DFT calculation of singly and doubly excited states, especially those highly excited Rydberg states that have not been achieved before within DFT. While in the present work we report results only for singly and doubly excited states, there are reasons to hope that this procedure should be equally accurate and applicable for other excitations, e.g., the triply and quadruply excited states, satellites, etc., as well.

The layout of the paper is as follows. Section II gives an outline of the formalism along with the numerical method employed. Section III presents a discussion on the results while Sec. IV makes a few concluding remarks.

\section{METHODOLOGY}

In this section, we first outline the work-function methodology for the excited states. Then we present the generalized pseudospectral method for the solution of the KS equation.

Assuming that a unique local exchange potential exists for a particular excited state, one can physically interpret $[17,18]$ it as the work required to move an electron against the electric field $\mathcal{E}_{x}(\mathbf{r})$ arising out of the Fermi hole charge distribution. The electric field, as such, is given by (atomic units employed unless otherwise mentioned)

$$
\mathcal{E}_{x}(\mathbf{r})=\int \frac{\rho_{x}\left(\mathbf{r}, \mathbf{r}^{\prime}\right)\left(\mathbf{r}-\mathbf{r}^{\prime}\right)}{\left|\mathbf{r}-\mathbf{r}^{\prime}\right|^{3}} d \mathbf{r} .
$$

The exchange potential with which the electrons move is then given by the line integral

$$
v_{x}(\mathbf{r})=-\int_{\infty}^{r} \mathcal{E}_{x}(\mathbf{r}) \cdot d \boldsymbol{l}
$$

The work $v_{x}(\mathbf{r})$ against the force field due to the Fermi hole charge can be determined exactly since the Fermi hole is known explicitly in terms of orbitals as

$$
\rho_{x}\left(\mathbf{r}, \mathbf{r}^{\prime}\right)=-\frac{\left|\gamma\left(\mathbf{r}, \mathbf{r}^{\prime}\right)\right|^{2}}{2 \rho(\mathbf{r})},
$$

where

$$
\gamma\left(\mathbf{r}, \mathbf{r}^{\prime}\right)=\sum_{i} \phi_{i}^{*}(\mathbf{r}) \phi_{i}\left(\mathbf{r}^{\prime}\right)
$$

Here $\gamma\left(\mathbf{r}, \mathbf{r}^{\prime}\right)$ is the single-particle density matrix spherically averaged over electronic coordinates for a given orbital angular quantum number, $\phi_{i}(\mathbf{r})=R_{n l}(r) Y_{l m}(\Omega)$ is the singleparticle orbital, and $\rho(\mathbf{r})$ is the total electron density expressed in terms of the occupied orbitals,

$$
\rho(\mathbf{r})=\sum_{i}\left|\phi_{i}(\mathbf{r})\right|^{2} .
$$

For spherically symmetric systems, Eq. (1) can be simplified as [18]

$$
\mathcal{E}_{x, r}(r)=-\frac{1}{4 \pi} \int \rho_{x}\left(\mathbf{r}, \mathbf{r}^{\prime}\right) \frac{\partial}{\partial r} \frac{1}{\left|\mathbf{r}-\mathbf{r}^{\prime}\right|} d \mathbf{r}^{\prime} d \Omega_{r} .
$$

Using the expansion

$$
\frac{1}{\left|\mathbf{r}-\mathbf{r}^{\prime}\right|}=4 \pi \sum_{l^{\prime \prime}, m^{\prime \prime}} \frac{1}{2 l^{\prime \prime}+1} Y_{l^{\prime \prime} m^{\prime \prime}}^{*}(\Omega) Y_{l^{\prime \prime} m^{\prime \prime}}\left(\Omega^{\prime}\right) \frac{r_{<}^{l^{\prime \prime}}}{r_{>}^{l^{\prime \prime}+1}},
$$

we obtain

$$
\begin{aligned}
\mathcal{E}_{x, r}(r)= & \frac{1}{2 \pi \rho(r)} \int \sum_{n, l, m, n^{\prime}, l^{\prime}, m^{\prime}, l^{\prime \prime}} R_{n l}(r) R_{n l}\left(r^{\prime}\right) R_{n^{\prime} l^{\prime}}(r) \\
& \times R_{n^{\prime} l^{\prime}}\left(r^{\prime}\right)\left[\frac{\partial}{\partial r} \frac{r_{<}^{l^{\prime \prime}}}{r_{>}^{l^{\prime \prime}+1}}\right] r^{\prime 2} d r^{\prime} \frac{(2 l+1)}{\left(2 l^{\prime}+1\right)} \\
& \times C^{2}\left(l l^{\prime \prime} l^{\prime} ; m, m^{\prime}-m, m^{\prime}\right) C^{2}\left(l l^{\prime \prime} l^{\prime} ; 000\right)
\end{aligned}
$$

where $R_{n l}(r)$ denotes the radial part of the single-particle orbitals and the $C$ 's are the Clebsch-Gordan coefficients [33]. Now the integral in Eq. (2) can be written as an integral over the radial coordinate only,

$$
v_{x}(r)=-\int_{\infty}^{r} \mathcal{E}_{x, r}\left(r^{\prime}\right) d r^{\prime} .
$$

While the exchange potential $v_{x}(\mathbf{r})$ can be accurately calculated, the correlation potential $v_{c}(\mathbf{r})$ is unknown and must be approximated. In the present calculation, we have used the generalized gradient-corrected correlation energy functional of Lee, Yang, and Parr (LYP) [34]. With this choice of $v_{x}(\mathbf{r})$ and $v_{c}(\mathbf{r})$, the following KS-type differential equation is solved:

$$
\left[-\frac{1}{2} \nabla^{2}+v_{e s}(\mathbf{r})+v_{x c}(\mathbf{r})\right] \phi_{i}(\mathbf{r})=\varepsilon_{i} \phi_{i}(\mathbf{r}),
$$

where $v_{e s}(\mathbf{r})$ is the usual Hartree electrostatic potential including electron-nuclear attraction and inter-electronic Coulomb repulsion, and $v_{x c}(\mathbf{r})=v_{x}(\mathbf{r})+v_{c}(\mathbf{r})$.

In this work, we extend the generalized pseudospectral method for the nonuniform and optimal spatial discretization and solution of the KS equation, Eq. (9). The procedure has been demonstrated to be capable of providing high precision solutions for the eigenvalues and wave functions for the study of electronic structure [29], multiphoton processes in strong fields [27,28,30,31], and Rydberg atom spectroscopy 
and dynamics [32]. In addition, the GPS method is computationally orders of magnitude faster than the equal-spacing FD methods. In the following, we briefly outline the GPS procedure appropriate for the present DFT study.

The main feature of the pseudospectral method is to approximate an exact function $f(x)$ defined on the interval $[-1,1]$ by the $N$ th-order polynomial $f_{N}(x)$,

$$
f(x) \cong f_{N}(x)=\sum_{j=0}^{N} f\left(x_{j}\right) g_{j}(x),
$$

and ensure the approximation to be exact at the collocation points $x_{j}$,

$$
f_{N}\left(x_{j}\right)=f\left(x_{j}\right)
$$

The present work employs the Legendre pseudospectral method where $x_{0}=-1, x_{N}=1$, and $x_{j}(j=1, \ldots, N-1)$ are determined by the roots of the first derivative of the Legendre polynomial $P_{N}(x)$ with respect to $x$, namely,

$$
P_{N}^{\prime}\left(x_{j}\right)=0 \text {. }
$$

In Eq. (10), $g_{j}(x)$ are the cardinal functions defined by

$$
g_{j}(x)=-\frac{1}{N(N+1) P_{N}\left(x_{j}\right)} \frac{\left(1-x^{2}\right) P_{N}^{\prime}(x)}{x-x_{j}},
$$

and they satisfy the unique property $g_{j}\left(x_{j^{\prime}}\right)=\delta_{j^{\prime} j}$.

The eigenvalue problem for the radial KS-type equation

$$
\hat{H}(r) \psi(r)=E \psi(r)
$$

with

$$
\hat{H}(r)=-\frac{1}{2} \frac{d^{2}}{d r^{2}}+V(r),
$$

for the structure and dynamics calculations involving a Coulomb potential typically has a singularity problem at $r=0$, and long-range $-1 / r$ behavior. This usually requires a large number of grid points in the equal-spacing finite-difference methods, which are not feasible to extend to Rydberg state calculations. This can be overcome by first mapping the semi-infinite domain $r \in[0, \infty]$ into the finite domain $x$ $\in[-1,1]$ by the mapping transformation $r=r(x)$ and then using the Legendre pseudospectral discretization technique. We use the following algebraic nonlinear mapping $[25,26]$ :

$$
r=r(x)=L \frac{1+x}{1-x+\alpha},
$$

where $\mathrm{L}$ and $\alpha=2 L / r_{\max }$ are the mapping parameters. Introducing

$$
\psi(r(x))=\sqrt{r^{\prime}(x)} f(x)
$$

and following the symmetrization procedure [25,26], we obtain the transformed Hamiltonian as
TABLE I. Comparison of the computed negative total energies and highest-occupied orbital energies of $\mathrm{He}, \mathrm{Be}, \mathrm{Ne}, \mathrm{Mg}$, and $\mathrm{Ar}$ (in a.u.) as obtained from the present $\mathrm{X}$-only calculation with the literature data. Numbers in parentheses denote the absolute percentage deviations relative to the literature values. HF and IE stand for Hartree-Fock and ionization energy, respectively.

\begin{tabular}{ccccc}
\hline \hline Atom & $-E(X$ only $)$ & $-E(\mathrm{HF})^{\mathrm{a}}$ & $\mathrm{IE}(\mathrm{X}$ only $)$ & $\mathrm{IE}(\mathrm{HF})^{\mathrm{a}}$ \\
\hline $\mathrm{He}$ & $2.8616(0.0035)$ & 2.8617 & $0.9180(0.0000)$ & 0.9180 \\
$\mathrm{Be}$ & $14.5726(0.0023)$ & 14.5730 & $0.3105(0.3880)$ & 0.3093 \\
$\mathrm{Ne}$ & $128.5434(0.0028)$ & 128.5471 & $0.8548(0.5127)$ & 0.8504 \\
$\mathrm{Mg}$ & $199.6096(0.0025)$ & 199.6146 & $0.2565(1.3830)$ & 0.2530 \\
$\mathrm{Ar}$ & $526.8062(0.0021)$ & 526.8174 & $0.5896(0.2369)$ & 0.5910 \\
\hline \hline
\end{tabular}

${ }^{\mathrm{a}}$ Reference [35].

$$
\hat{H}(x)=-\frac{1}{2} \frac{1}{r^{\prime}(x)} \frac{d^{2}}{d x^{2}} \frac{1}{r^{\prime}(x)}+V(r(x))+V_{m}(x),
$$

where

$$
V_{m}(x)=\frac{3\left(r^{\prime \prime}\right)^{2}-2 r^{\prime \prime \prime} r^{\prime}}{8\left(r^{\prime}\right)^{4}}
$$

This leads to a symmetric matrix eigenvalue problem. Note that for the mapping used in Eq. (16) $V_{m}(x)=0$. Therefore discretizing the Hamiltonian by the generalized pseudospectral method leads to the following set of coupled equations:

$$
\begin{gathered}
\sum_{j=0}^{N}\left[-\frac{1}{2} D_{j^{\prime} j}^{(2)}+\delta_{j^{\prime} j} V\left(r\left(x_{j}\right)\right)+\delta_{j^{\prime} j} V_{m}\left(r\left(x_{j}\right)\right)\right] A_{j}=E A_{j^{\prime}} \\
j=1, \ldots, N-1, \\
A_{j}=r^{\prime}\left(x_{j}\right) f\left(x_{j}\right)\left[P_{N}\left(x_{j}\right)\right]^{-1} \\
=\left[r^{\prime}\left(x_{j}\right)\right]^{1 / 2} \psi\left(r\left(x_{j}\right)\right)\left[P_{N}\left(x_{j}\right)\right]^{-1} .
\end{gathered}
$$

Here $D_{j^{\prime} j}^{(2)}$ represents the symmetrized second derivative of the cardinal function with respect to $r$,

$$
D_{j^{\prime} j}^{(2)}=\left[r^{\prime}\left(x_{j^{\prime}}\right)\right]^{-1} d_{j^{\prime} j}^{(2)}\left[r^{\prime}\left(x_{j}\right)\right]^{-1},
$$

and

$$
d_{j^{\prime}, j}^{(2)}= \begin{cases}\frac{1}{r^{\prime}(x)} \frac{(N+1)(N+2)}{6\left(1-x_{j}\right)^{2}} \frac{1}{r^{\prime}(x)}, & j=j^{\prime}, \\ \frac{1}{r^{\prime}\left(x_{j^{\prime}}\right)} \frac{1}{\left(x_{j}-x_{j^{\prime}}\right)^{2}} \frac{1}{r^{\prime}\left(x_{j}\right)}, & j \neq j^{\prime} .\end{cases}
$$

Equations (1) and (2) dictate the exchange potential for a particular atomic electronic configuration. Equation (9) is then numerically solved to achieve a self-consistent set of orbitals, using the GPS method. These orbitals are used to construct various Slater determinants arising out of that particular electronic configuration and its energies computed in the usual manner. If $E\left(D_{i}\right)$ and $E\left(M_{i}\right)$ denote the energies of 
TABLE II. Calculated singly excited triplet state energies of $\mathrm{He}$ (in a.u.) along with literature data for comparison. Numbers in parentheses denote the absolute percentage errors with respect to the literature data.

\begin{tabular}{|c|c|c|c|}
\hline State & $-E$ (X only) & $-E(\mathrm{XC})$ & $-E(\text { literature })^{\mathrm{a}}$ \\
\hline $1 s 2 s{ }^{3} S$ & $\begin{array}{c}2.17420 \\
2.17425^{\mathrm{b}}\end{array}$ & $2.17545(0.0101)$ & 2.17523 \\
\hline $1 s 3 s^{3} S$ & 2.06793 & $2.06890(0.0102)$ & 2.06869 \\
\hline $1 s 4 s^{3} S$ & 2.03606 & $2.03671(0.0098)$ & 2.03651 \\
\hline $1 s 5 s^{3} S$ & 2.02242 & $2.02264(0.0010)$ & 2.02262 \\
\hline $1 s 6 s^{3} S$ & 2.01530 & $2.01539(0.0010)$ & 2.01537 \\
\hline $1 s 7 s^{3} S$ & 2.01107 & $2.01115(0.0010)$ & 2.01113 \\
\hline $1 s 8 s^{3} S$ & 2.00840 & $2.00845(0.0010)$ & 2.00843 \\
\hline $1 s 9 s^{3} S$ & 2.00658 & $2.00660(0.0000)$ & 2.00660 \\
\hline $1 s 10 s^{3} S$ & 2.00530 & $2.00531(0.0000)$ & 2.00531 \\
\hline $1 s 11 s^{3} S$ & 2.00431 & $2.00431(0.0000)$ & 2.00431 \\
\hline $1 s 12 s^{3} S$ & 2.00365 & $2.00365(0.0000)$ & 2.00365 \\
\hline $1 s 13 s^{3} S$ & 2.00310 & $2.00310(0.0000)$ & 2.00310 \\
\hline $1 s 14 s^{3} S$ & 2.00266 & $2.00266(0.0000)$ & 2.00266 \\
\hline $1 s 15 s^{3} S$ & 2.00231 & $2.00231(0.0000)$ & 2.00231 \\
\hline $1 s 16 s^{3} S$ & 2.00203 & 2.00203 & \\
\hline
\end{tabular}

${ }^{\text {a }}$ Reference [36].

${ }^{b} \mathrm{HF}$ result, Ref. [37].

different determinants and multiplets, respectively, corresponding to a given configuration, then multiplet energies are calculated by following the diagonal sum rule $[8,19-23]$

$$
E\left(M_{j}\right)=\sum_{i} A_{j i} E\left(D_{i}\right)
$$

As an example, consider the calculation of individual multiplets corresponding to the nsnp configuration, which has associated with it 12 different determinants for the three different values $(+1,0,-1)$ of $\Sigma m_{l}$ and $\Sigma m_{s}$. Of these, there is a single determinant corresponding to $\sum m_{l}=\sum m_{s}=1$; this is the ${ }^{3} P$ term. There are two determinants for $\sum m_{l}=1$ and $\sum m_{s}=0$. The sum of these two determinants' energy equals the sum of the ${ }^{3} P$ and ${ }^{1} P$ terms. Hence the energy of the ${ }^{1} P$ term can be obtained by simply subtracting the ${ }^{3} P$ term energy from the above sum.

Two sets of calculations are performed for the singly excited states; the solution of Eq. (9) with $v_{x c}=v_{x}$ and $v_{x c}$ $=v_{x}+v_{c}\left(v_{L Y P}\right)$. For the doubly excited states we present only the second set. The convergence criteria for the potential and energy are $10^{-6}$ and $10^{-8}$ a.u., respectively. In our calculations, up to 500 radial grid points are used to achieve convergence.

\section{RESULTS AND DISCUSSION}

First we make a comparison of our ground state exchange- (X-)only results with the Hartree-Fock (HF) theory. Nonrelativistic ground state energies and highestoccupied orbital energies are presented for $\mathrm{He}, \mathrm{Be}, \mathrm{Ne}, \mathrm{Mg}$, and Ar along with the HF data [35] in Table I. The numbers
TABLE III. Calculated singly excited state energies of Li and Be (in a.u.) along with the literature data for comparison. Numbers in parentheses denote the absolute percentage deviations with respect to the literature data.

\begin{tabular}{cccc}
\hline \hline State & $-E($ X only $)$ & $-E($ XC $)$ & $-E($ literature $)$ \\
\hline & & $\mathrm{Li}$ & \\
$1 s^{2} 3 s^{2} S$ & 7.30966 & $7.35773(0.0515)$ & $7.35394^{\mathrm{b}}$ \\
& $7.31021^{\mathrm{a}}$ & & \\
$1 s^{2} 4 s^{2} S$ & 7.27466 & $7.31978(0.0193)$ & $7.31837^{\mathrm{b}}$ \\
$1 s^{2} 5{ }^{2} S$ & 7.25996 & $7.30466(0.0130)$ & $7.30339^{\mathrm{b}}$ \\
$1 s^{2} 2 p^{2} P$ & 7.36486 & $7.41204(0.0254)$ & $7.41016^{\mathrm{c}}$ \\
& $7.36507^{\mathrm{a}}$ & & \\
$1 s^{2} 3 p^{2} P$ & 7.29295 & $7.33862(0.0199)$ & $7.33716^{\mathrm{c}}$ \\
& $7.29319^{\mathrm{a}}$ & & \\
$1 s^{2} 4 p^{2} P$ & 7.26859 & $7.31262(0.0098)$ & $7.31190^{\mathrm{c}}$ \\
$1 s^{2} 5 p^{2} P$ & 7.25756 & $7.30053(0.0031)$ & $7.30030^{\mathrm{c}}$ \\
& & & \\
$1 s^{2} 2 s 3 s^{3} S$ & 14.37798 & $14.42917(0.1996)$ & $14.42629^{\mathrm{e}}$ \\
$1 s^{2} 2 s 4 s^{3} S$ & 14.32506 & 14.37090 & \\
$1 s^{2} 2 s 5 s^{3} S$ & 14.30562 & 14.34996 & \\
$1 s^{2} 2 s 2 p^{3} P$ & 14.51068 & $14.56660(0.0300)$ & $14.56223^{\mathrm{e}}$ \\
& $14.51150^{\mathrm{d}}$ & & \\
$1 s^{2} 2 s 3 p^{3} P$ & 14.34826 & $14.39797(0.0199)$ & $14.39510^{\mathrm{e}}$ \\
$1 s^{2} 2 s 4 p^{3} P$ & $14.34886^{\mathrm{d}}$ & & \\
$1 s^{2} 2 s 5 p^{3} P$ & 14.31462 & 14.35910 & \\
\hline \hline
\end{tabular}

${ }^{\mathrm{a}} \mathrm{HF}$ result, Ref. [38].

${ }^{\mathrm{b}}$ Reference [39].

${ }^{\mathrm{c}}$ Reference [40].

${ }^{\mathrm{d}} \mathrm{HF}$ result, Ref. [41].

${ }^{\mathrm{e}}$ Reference [42].

in parentheses denote the absolute percentage deviations with respect to the HF results. While the total ground state energies have never fallen below the HF results, the ionization potentials show both positive and negative deviations. The agreement of the calculated results with the HF values is generally excellent. The deviations in energy range from $0.0021 \%$ to $0.0035 \%$, whereas those for the ionization potentials go from $0.000 \%$ to $1.383 \%$.

Considerable attention has been paid by both experimentalists and theoreticians (using ab initio multiconfiguration interaction methods) to the study of the excited states of many-electron systems, especially for low- $Z$ atomic systems. Some of these literature data will be used to calibrate the accuracy of our results. As the present X-only results are quite accurate and should be comparable to the HF results, to put our results in proper perspective, for singly excited states, we have presented the individual state energies computed from two sets of calculations, namely, the X-only and with both exchange and correlation included, with the aim of isolating the errors generated by the approximation in the correlation functional. However, for the doubly excited states studied in this work, we could not find suitable literature data for direct comparison with the $\mathrm{X}$-only results; accordingly they are 
TABLE IV. Calculated doubly excited state $\left(n s^{2}, n s n^{\prime} p\right)$ energies of He (in a.u.) along with literature data for comparison. Numbers in parentheses denote the absolute percentage errors with respect to the literature data.

\begin{tabular}{|c|c|c|}
\hline State & $-E(\mathrm{XC})$ & $-E($ literature $)$ \\
\hline $2 s^{2}{ }^{1} S$ & $0.76637(1.48)$ & $0.77787^{\mathrm{a}}$ \\
\hline $3 s^{2}{ }^{1} S$ & $0.34578(2.19)$ & $0.35354^{\mathrm{a}}$ \\
\hline $4 s^{21} S$ & $0.19659(2.19)$ & $0.20099^{\mathrm{a}}$ \\
\hline $5 s^{21} S$ & $0.12754(2.12)$ & $0.13030^{\mathrm{b}}$ \\
\hline $6 s^{2}{ }^{1} S$ & $0.08808(3.05)$ & $0.09085^{\mathrm{b}}$ \\
\hline $7 s^{2}{ }^{1} S$ & $0.06524(3.35)$ & $0.0675^{\mathrm{b}}$ \\
\hline $8 s^{2}{ }^{1} S$ & 0.04855 & \\
\hline $9 s^{21} S$ & 0.03889 & \\
\hline $10 s^{21} s$ & 0.03084 & \\
\hline $11 s^{21} S$ & 0.02503 & \\
\hline $12 s^{21} S$ & 0.02121 & \\
\hline $13 s^{21} S$ & 0.01811 & \\
\hline $14 s^{21} S$ & 0.01555 & \\
\hline $15 s^{21} S$ & 0.01348 & \\
\hline $16 s^{21} S$ & 0.01176 & \\
\hline $17 s^{21} S$ & 0.01132 & \\
\hline $2 s 3 s^{3} S$ & $0.60056(0.34)$ & $0.60258^{\mathrm{a}, \mathrm{c}}$ \\
\hline $2 s 3 s^{1} S$ & $0.58328(1.12)$ & $0.58989^{\mathrm{a}}$ \\
\hline $3 s 4 s^{3} S$ & $0.28282(1.55)$ & $0.28728^{\mathrm{a}}$ \\
\hline $3 s 4 s^{1} S$ & $0.27302(2.86)$ & $0.28107^{\mathrm{a}}$ \\
\hline $4 s 5 s^{3} S$ & $0.16324(3.59)$ & $0.16931^{\mathrm{a}}$ \\
\hline $4 s 5 s^{1} S$ & $0.16007(3.41)$ & $0.16573^{\mathrm{a}}$ \\
\hline $5 s 6 s^{3} S$ & 0.10721 & \\
\hline $5 s 6 s^{1} S$ & 0.10277 & \\
\hline $2 s 2 p^{3} P$ & $0.76770(0.95)$ & $0.76049^{d}$ \\
\hline $2 s 3 p^{3} P$ & 0.58253 & $0.58467(+)^{\mathrm{d}}, 0.57903(-)^{\mathrm{d}}$ \\
\hline $2 s 4 p^{3} P$ & 0.54558 & $0.54283(+)^{\mathrm{d}}, 0.53956(-)^{\mathrm{d}}$ \\
\hline $2 s 5 p^{3} P$ & 0.53121 & $0.52571(+)^{\mathrm{d}}, 0.52394(-)^{\mathrm{d}}$ \\
\hline $2 s 6 p^{3} P$ & 0.52367 & $0.51211(+)^{\mathrm{d}}, 0.51608(-)^{\mathrm{d}}$ \\
\hline $2 s 7 p^{3} P$ & 0.51924 & $0.51219(+)^{\mathrm{d}}, 0.51155(-)^{\mathrm{d}}$ \\
\hline $3 s 3 p^{3} P$ & $0.34918(0.34)$ & $0.35038^{\mathrm{d}}$ \\
\hline $3 s 4 p^{3} P$ & 0.27365 & $0.27948(+)^{\mathrm{d}}, 0.26023(-)^{\mathrm{d}}$ \\
\hline $4 s 4 p^{3} P$ & $0.19787(1.11)$ & $0.2001^{\mathrm{e}}$ \\
\hline $4 s 5 p^{3} P$ & 0.15933 & \\
\hline
\end{tabular}

${ }^{\mathrm{a}}$ Reference [36].

${ }^{\mathrm{b}}$ Reference [42].

${ }^{\mathrm{c}}$ Reference [43].

${ }^{\mathrm{d}}$ Reference [44].

${ }^{\mathrm{e}}$ Reference [45].

omitted, and only the $\mathrm{XC}$ results will be presented. Comparisons have been made with the best available experimental and theoretical results, as well as with the HF data, wherever possible.

Table II reports the results for the $1 \operatorname{sns}(n=2-16)$ triplet $S$ states of He. For $1 s 2 s^{3} S$ state, the X-only result is -2.17420 a.u., only $0.0023 \%$ above the $\mathrm{HF}$ value of -2.17425 a.u. [37], indicating the accuracy of our calculations. For all the states, the present results with both exchange and correlation included match very closely the more sophisticated complex rotation calculations [36]. We note
TABLE V. Comparison of the calculated doubly excited state $\left(n p^{2}\right)$ energies of $\mathrm{He}$ (in a.u.) along with literature data. Numbers in parentheses denote the absolute percentage errors with respect to the literature data.

\begin{tabular}{|c|c|c|}
\hline State & $-E(\mathrm{XC})$ & $-E$ (literature $)$ \\
\hline $2 p^{2}{ }^{1} D$ & $0.69272(1.31)$ & $0.70195^{\mathrm{a}}$ \\
\hline $2 p^{23} P$ & $0.70626(0.60)$ & $0.71050^{\mathrm{a}}$ \\
\hline $3 p^{2}{ }^{1} D$ & $0.31540(0.04)$ & $0.31554^{\mathrm{a}}$ \\
\hline $3 p^{23} P$ & $0.33086(1.56)$ & $0.33609^{\mathrm{a}}$ \\
\hline $4 p^{2}{ }^{1} D$ & 0.18095 & \\
\hline $4 p^{23} P$ & 0.18353 & \\
\hline $5 p^{2}{ }^{1} D$ & 0.11610 & \\
\hline $5 p^{23} P$ & 0.11764 & \\
\hline $6 p^{21} D$ & 0.08115 & \\
\hline $6 p^{23} P$ & 0.08231 & \\
\hline $7 p^{21} D$ & 0.05980 & \\
\hline $7 p^{23} P$ & 0.06040 & \\
\hline $8 p^{2}{ }^{1} D$ & 0.04565 & \\
\hline $8 p^{23} P$ & 0.04620 & \\
\hline $9 p^{2}{ }^{1} D$ & 0.03604 & \\
\hline $9 p^{23} P$ & 0.03650 & \\
\hline $10 p^{21} D$ & 0.02920 & \\
\hline $10 p^{23} P$ & 0.02967 & \\
\hline $11 p^{21} D$ & 0.02414 & \\
\hline $11 p^{23} P$ & 0.02454 & \\
\hline $12 p^{21} D$ & 0.02028 & \\
\hline $12 p^{23} P$ & 0.02067 & \\
\hline $13 p^{21} D$ & 0.01728 & \\
\hline $13 p^{23} P$ & 0.01764 & \\
\hline $14 p^{21} D$ & 0.01490 & \\
\hline $14 p^{23} P$ & 0.01523 & \\
\hline $15 p^{21} D$ & 0.01297 & \\
\hline $15 p^{23} P$ & 0.01329 & \\
\hline $16 p^{21} D$ & 0.01140 & \\
\hline $16 p^{23} P$ & 0.01170 & \\
\hline $17 p^{21} D$ & 0.01010 & \\
\hline $17 p^{23} P$ & 0.01039 & \\
\hline
\end{tabular}

${ }^{\mathrm{a}}$ Reference [43].

that for the high-lying Rydberg states $(n>10)$, the results of the three columns ( $\mathrm{X}$ only, $\mathrm{XC}$, and the best available literature data) are essentially identical. This is consistent with the fact that for Rydberg states the asymptotic long-range Coulomb potential (arising solely from the exchange interaction) is the dominant factor for electronic structure determination, and the electron correlation plays little role here. As $n$ decreases, the deviations between the three results increase, since the electron correlation now plays an increasingly important role. For low $n$, our XC results fall slightly below the literature data, presumably because of the overestimation caused by the LYP correlation energy functional. However, in all cases, the discrepancy is no more than $0.0102 \%$. It is gratifying that the present single-determinantal results are comparable to other more sophisticated $a b$ initio calculations. Also, as $n$ increases, the spacings between the states decrease and the present method reproduces that very well, a 
TABLE VI. Comparison of the calculated single- and double-excitation energies of $\mathrm{He}$ and $\mathrm{Be}$ (in a.u.) along with the literature data. Numbers in parentheses denote the absolute percentage errors with respect to the best theoretical data.

\begin{tabular}{|c|c|c|c|c|}
\hline State & $\begin{array}{c}\text { Present } \\
\text { work }\end{array}$ & $\Delta \epsilon_{K S}$ & $\begin{array}{l}\text { Other } \\
\text { theory }\end{array}$ & Experiment \\
\hline \multicolumn{5}{|c|}{ Single excitation of $\mathrm{He}$ and $\mathrm{Be}$} \\
\hline He $1 s 2 s^{3} S$ & $0.72839(0.015)$ & $0.7460^{\mathrm{a}}$ & $0.72850^{\mathrm{b}}$ & $0.72833^{\mathrm{c}}$ \\
\hline $\mathrm{He} 1 s 2 s{ }^{1} S$ & $0.75759(0.021)$ & & $0.75775^{\mathrm{b}}$ & $0.75759^{c}$ \\
\hline He $1 s 2 p^{3} P$ & $0.77041(0.019)$ & $0.7772^{\mathrm{a}}$ & $0.77056^{\mathrm{b}}$ & $0.77039^{c}$ \\
\hline $\mathrm{He} 1 s 2 p{ }^{1} P$ & $0.77971(0.022)$ & & $0.77988^{\mathrm{b}}$ & $0.77972^{\mathrm{c}}$ \\
\hline He $1 s 3 s^{3} S$ & $0.83494(0.012)$ & $0.8392^{\mathrm{a}}$ & $0.83504^{\mathrm{b}}$ & $0.83486^{\mathrm{c}}$ \\
\hline $\mathrm{He} 1 s 3 s{ }^{1} S$ & $0.84231(0.017)$ & & $0.84245^{\mathrm{b}}$ & $0.84228^{\mathrm{c}}$ \\
\hline $\mathrm{He} 1 s 3 p^{3} P$ & $0.84548(0.020)$ & $0.8476^{\mathrm{a}}$ & $0.84564^{\mathrm{b}}$ & $0.84547^{\mathrm{c}}$ \\
\hline $\mathrm{He} 1 s 3 p{ }^{1} P$ & $0.84841(0.020)$ & & $0.84858^{\mathrm{b}}$ & $0.84841^{\mathrm{c}}$ \\
\hline $\mathrm{He} 1 s 4 s^{3} S$ & $0.86713(0.009)$ & $0.8688^{\mathrm{a}}$ & $0.86721^{\mathrm{b}}$ & $0.86704^{\mathrm{c}}$ \\
\hline He $1 s 4 s{ }^{1} S$ & $0.87003(0.013)$ & & $0.87014^{\mathrm{b}}$ & $0.86997^{\mathrm{c}}$ \\
\hline Be $1 s^{2} 2 s 2 p^{3} P$ & 0.10089 & $0.1327^{\mathrm{a}}$ & & $0.100153^{\mathrm{c}}$ \\
\hline Be $1 s^{2} 2 s 3 s^{3} S$ & $0.23832(0.632)$ & $0.2444^{\mathrm{a}}$ & $0.236823^{\mathrm{d}}$ & $0.237304^{\mathrm{c}}$ \\
\hline Be $1 s^{2} 2 s 3 p^{3} P$ & 0.26952 & $0.2694^{\mathrm{a}}$ & & $0.267877^{\mathrm{c}}$ \\
\hline $\mathrm{Be} 1 s^{2} 2 s 4 s^{3} S$ & 0.29659 & $0.2959^{\mathrm{a}}$ & & $0.293921^{\mathrm{c}}$ \\
\hline Be $1 s^{2} 2 s 4 p^{3} P$ & 0.30839 & $0.3046^{\mathrm{a}}$ & & $0.300487^{\mathrm{c}}$ \\
\hline Be $1 s^{2} 2 s 5 s^{3} S$ & 0.31753 & $0.3153^{\mathrm{a}}$ & & $0.314429^{c}$ \\
\hline \multicolumn{5}{|c|}{ Double excitation of $\mathrm{He}$} \\
\hline He $2 s^{2}{ }^{1} S$ & $2.13747(0.544)$ & & $2.1259^{\mathrm{e}}, 2.1285^{\mathrm{f}}$ & \\
\hline $\mathrm{He} 3 s^{2}{ }^{1} S$ & $2.55806(0.612)$ & & $2.5425^{\mathrm{g}}, 2.5496^{\mathrm{f}}$ & \\
\hline $\mathrm{He} 4 s^{2}{ }^{1} S$ & $2.70725(0.484)$ & & $2.6942^{\mathrm{g}}, 2.7017^{\mathrm{f}}$ & \\
\hline $\mathrm{He} 5 s^{2}{ }^{1} S$ & $2.77630(0.101)$ & & $2.7735^{\mathrm{g}}$ & \\
\hline He $6 s^{2}{ }^{1} S$ & $2.81576(0.102)$ & & $2.8129^{\mathrm{g}}$ & \\
\hline $\mathrm{He} 7 s^{2}{ }^{1} S$ & $2.83860(0.085)$ & & $2.8362^{\mathrm{h}}$ & \\
\hline He $2 p^{2}{ }^{1} D$ & $2.21120(0.684)$ & & $2.1961^{\mathrm{h}}, 2.2082^{\mathrm{f}}$ & \\
\hline He $3 p^{2}{ }^{1} D$ & $2.58844(1.600)$ & & $2.5477^{\mathrm{h}}, 2.5595^{\mathrm{f}}$ & \\
\hline $\mathrm{He} 4 p^{2}{ }^{1} D$ & $2.72289(1.080)$ & & $2.6938^{h}$ & \\
\hline He $2 s 3 s^{3} S$ & $2.30328(0.095)$ & & $2.3011^{\mathrm{i}}$ & \\
\hline $\mathrm{He} 2 s 3 s^{1} S$ & $2.32056(0.292)$ & & $2.3138^{\mathrm{i}}, 2.3194^{\mathrm{g}}$ & $2.3130^{\mathrm{j}}$ \\
\hline He $3 s 4 s{ }^{3} S$ & $2.62102(0.177)$ & & $2.6164^{\mathrm{i}}$ & \\
\hline $\mathrm{He} 3 s 4 s{ }^{1} S$ & $2.63082(0.310)$ & & $2.6227^{\mathrm{i}}, 2.6249^{\mathrm{g}}$ & \\
\hline $\mathrm{He} 4 s 5 s^{3} S$ & $2.74060(0.227)$ & & $2.7344^{\mathrm{i}}$ & \\
\hline He $4 s 5 s{ }^{1} S$ & $2.74377(0.211)$ & & $2.7380^{\mathrm{i}}, 2.7385^{\mathrm{g}}$ & \\
\hline
\end{tabular}

${ }^{\mathrm{a}}$ Reference [5].

${ }^{\mathrm{b}}$ References [49,50].

${ }^{\mathrm{c}}$ Reference [51].

${ }^{\mathrm{d}}$ Reference [52].

${ }^{\mathrm{e}}$ Reference [53].

feature that was noticed earlier for core excitations and satellites $[21,22]$ as well. It may be mentioned that for highly excited state calculations with large $n$, many quantum mechanical calculations encounter a common problem of selfconsistent convergence. However, in our calculations, we got converged results for all the states with the same set of grid parameters, including the number of points, reflecting the accuracy in the exchange potential and the GPS procedure employed. Also although we presented here results for $n$ $=16$, it is straightforward to extend to even higher states. Finally, even though the method is nonvariational, there is no anomaly in the ordering of the closely spaced energy levels.
${ }^{\mathrm{f}}$ Reference [54].
${ }^{\mathrm{g}}$ Reference [42].
${ }^{\mathrm{h}}$ Reference [55].
${ }^{\mathrm{i}}$ Reference [36].
${ }^{\mathrm{j}}$ Reference [56].

Now we present results for more than two-electron atoms. Table III presents results for the singly excited $1 s^{2} n s^{2} S$ and $1 s^{2} n p^{2} P$ states of $\mathrm{Li}$ as well as the $1 s^{2} 2 s n s^{3} S$ and $1 s^{2} 2 s 2 p^{3} P$ states of Be. Again, the $\mathrm{X}$-only results are fairly close to the HF results, errors ranging from $0.0057 \%$ to as low as $0.0001 \%$ for Be $1 s^{2} 2 s 4 p^{3} P$. The Li doublet $S$ states are quoted from the full-core-plus-correlation method with multiconfiguration interaction wave functions [39], while the doublet $P$ states are quoted from the combined configuration-interaction-Hylleraas method [40]. For Be, the literature data are quite scanty and the density-functional results compare quite satisfactorily with the multiconfiguration 
calculations [42]. As earlier, there is some overestimation in the total energy caused by the LYP correlation potential. The errors range from $0.0515 \%$ to $0.0031 \%$. Several states are reported here for the first time to our knowledge.

Next we present results for the doubly excited states for He. Table IV displays results for the doubly excited $n s^{2}{ }^{1} S^{e}$ $(n=2-16), n s n^{\prime} s^{3} S^{e},{ }^{1} S^{e} \quad\left(n=2-5, \quad n^{\prime}=3-6, \quad n \neq n^{\prime}\right)$, $n s n^{\prime} p^{3} P^{o}\left(n=2, n^{\prime}=2-7 ; n=3,4, n^{\prime}=n, n+1\right)$ states of $\mathrm{He}$, while Table $\mathrm{V}$ presents results for $n p^{23} P^{e},{ }^{1} D^{e}(n$ $=2-17)$ states of He. Many of these states have been identified as autoionizing in the literature, e.g., the $n s^{2}{ }^{1} S^{e}$ states. Except for the case of $2 s 2 p^{3} P^{o}$, the calculated energies for the doubly excited states never fall below the quoted results. In contrast to the singly excited Rydberg states for $\mathrm{He}$, the results for the doubly excited $n s^{2}$ states are comparable to the literature data for smaller $n$ and the discrepancy tends to increase as $n$ increases. This may occur because of the errors in the long-range behavior of the correlation potential or the work-function formalism itself. For the $n s n^{\prime} s^{3} S^{e},{ }^{1} S^{e}$ and $n s n^{\prime} p^{3} P^{o}$ states, our density-functional results are seen to be satisfactory. It may be noted that $n s n^{\prime} s^{1} S^{e}$ states with $n \neq n^{\prime}$ cannot be represented by a single Slater determinant and these states have been calculated by following a procedure similar to Slater's diagonal sum rule [19-23; see Eq. (24)]. For the odd-parity $n s n^{\prime} p$ triplet states with $n \neq n^{\prime}$, literature results are available in the form of $a(+,-)$ classification [46], signifying the relative (in or out of phase) radial motion of the two electrons. However, the present scheme does not separately identify the $(+)$ and $(-)$ states and the reported results for these states may be considered as an arithmetic mean of the two. For the even-parity ${ }^{1} D$ and ${ }^{3} P$ states arising from the $n p^{2}$ configuration, literature data are quite scarce, and no results are available for high $n$. From Tables IV and V, we see that, while the accuracy of our doubly excited state calculations is not as good as that of singly excited states, the error for the former is still well within $3.6 \%$ overall.

Table VI presents results for the single- and doubleexcitation energies for 31 selected states along with the literature data. These are calculated with respect to the present
TABLE VII. Calculated single-particle expectation values (in a.u.) for some of the excited states of $\mathrm{He}$ and $\mathrm{Be}$ along with the literature data for comparison. In the last four columns, the first and second entries for a particular state correspond to the X-only and the $\mathrm{XC}$ results, respectively.

\begin{tabular}{lcccc}
\hline \hline \multicolumn{1}{c}{ State } & $\left\langle 1 / r^{2}\right\rangle$ & $\langle 1 / r\rangle$ & $\langle r\rangle$ & $\left\langle r^{2}\right\rangle$ \\
\hline He $1 s 2 s{ }^{3} S$ & 4.1725 & 1.1573 & 2.5066 & 11.0469 \\
& 4.2197 & 1.1668 & 2.4502 & 10.4777 \\
He $1 s 16{ }^{3} S$ & & $1.1544^{\mathrm{a}}$ & $2.5599^{\mathrm{a}}$ & $11.5612^{\mathrm{a}}$ \\
& 4.0077 & 1.0031 & 174.190 & 67471.45 \\
He $2 s 3 s^{3} S$ & 4.0453 & 1.0087 & 168.872 & 63581.07 \\
& 0.56104 & 0.32538 & 5.9366 & 49.5937 \\
Li $1 s^{2} 3 s^{2} S$ & 0.57400 & 0.33026 & 5.7952 & 46.8985 \\
& 9.9772 & 1.8393 & 3.6141 & 36.0944 \\
Be $1 s^{2} 2 s 2 p{ }^{3} P$ & 10.0323 & 1.8466 & 3.5232 & 34.0835 \\
& 14.2006 & 2.0933 & 1.5222 & 4.3390 \\
& 14.2442 & 2.1006 & 1.4940 & 4.1436 \\
& & $2.0882^{\mathrm{a}}$ & $1.5626^{\mathrm{a}}$ & $4.5884^{\mathrm{a}}$ \\
\hline \hline
\end{tabular}

${ }^{\mathrm{a}} \mathrm{HF}$ result, Ref. [37].

ground state energies of -2.90384 and -14.66749 a.u., for $\mathrm{He}$ and $\mathrm{Be}$, respectively. Experimental results are quite scarce for double excitations. It is noticed that, for some cases, the calculated excitation energies fall below the experimental result. The present method is nonvariational, and consequently the variational restriction on the excited state being the lowest state of a given space-spin symmetry does not apply. The table also shows the single-electron KohnSham energies (obtained from the differences of Kohn-Sham eigenvalues) for single excitations for $\mathrm{He}$ and $\mathrm{Be}$ [5]. Of course, the single-electron Kohn-Sham energies do not distinguish the multiplets. As observed, the excitation energies from the true Kohn-Sham potential for $\mathrm{He}$ and $\mathrm{Be}$ are quite good. However, it may be mentioned that the excitation energies obtained from other commonly used approximate density functionals [such as the local density approximation (LDA)] have produced large errors ([5]; note that both the LDA and Becke [47] exchange potentials produced large er-

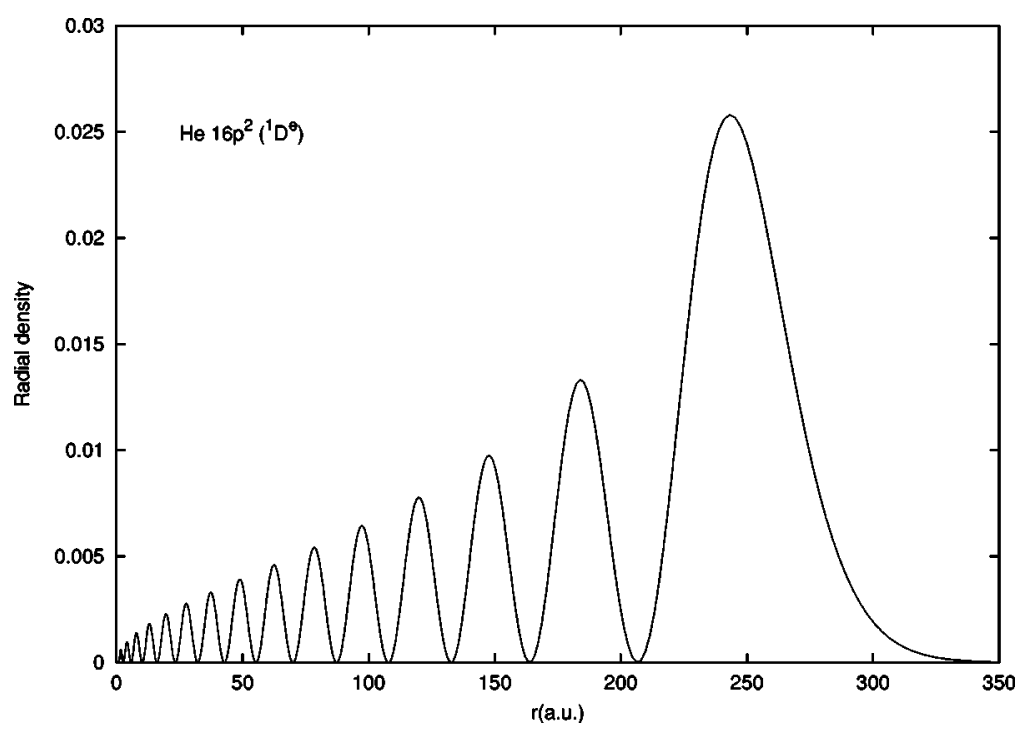

FIG. 1. Radial density (in a.u.) plot for $\mathrm{He}$ $16 s^{2} S^{e}$ state. 


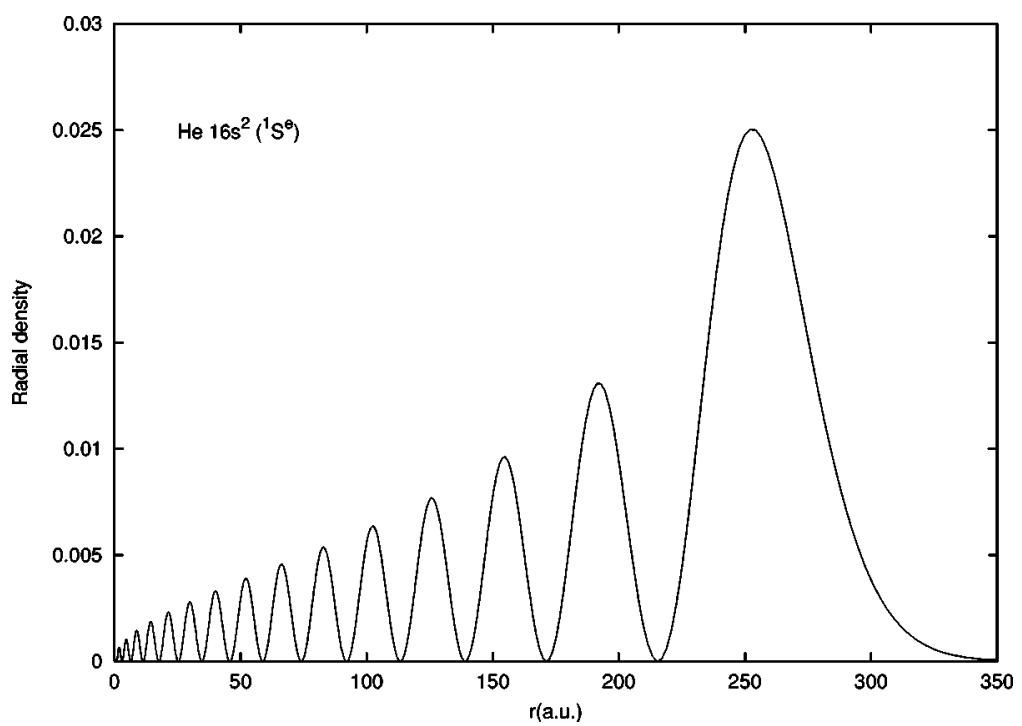

FIG. 2. Radial density (in a.u.) plot for $\mathrm{He}$ $16 p^{21} D^{e}$ state. rors in excitation energy for Ne satellites [22], making them quite unsuitable for excited state calculations). It is also to be noted that the present calculation is nonrelativistic and the experimental results, of course, include relativistic effects. Good agreement is observed for the single-excitation energies of He with both theoretical and experimental results. For $\mathrm{Be}$, the discrepancies are usually higher than those for He. But nevertheless there is satisfactory agreement between the present results and the literature data. A part of this error may be attributed to the inefficiency of the present singledeterminantal approach in dealing with the electron correlation using the LYP correlation functional. It could also be rooted in the assumption of spherical symmetry in calculating the exchange potential. Put differently, the solenoidal component of the electric field $\mathcal{E}_{x}(\mathbf{r})$ may not be negligible in comparison to the irrotational component for these states, although this usually holds true for atoms [48].

Finally, some representative expectation values are presented along with the literature data in Table VII. As is seen, they match nicely with the other results. Also Figs. 1 and 2 depict the calculated radial densities for the $16 s^{2}{ }^{1} S^{e}$ and $16 p^{2}{ }^{1} D^{e}$ states of He. As expected, there are 16 maxima (the first peak can be seen after magnification) for the $16 s^{2}$ and 15 maxima for the $16 p^{2}$ configurations.

\section{CONCLUSION}

There have been many attempts $[3,6]$ to calculate excited states of many-electron systems within DFT. Various attractive formalisms have been proposed; some of them are computationally difficult to implement and others produce large errors. In addition, it is not a straightforward task to extract the radial densities. Also, all the attempts so far have been restricted to single excitations. To the best of our knowledge, no results have been reported for multiple excitations so far except using the work-function formalism. The present methodology provides reasonably good results for the excited state energy, excitation energy, and radial densities for both single and multiple atomic excitations including the highlying states.

About 100 low- and high-lying singly excited states of $\mathrm{He}, \mathrm{Li}$, and $\mathrm{Be}$ and doubly excited states of He have been presented by using the work-function formalism within the nonrelativistic DFT framework. The numerical solution of the resulting KS equation by the accurate GPS method provides good results for the excited state energy, excitation energy, radial density, and expectation values for both single and multiple atomic excitations including the high-lying states. The single-determinantal approach offers nearly-HFquality results for the $\mathrm{X}$-only case, and inclusion of the nonlinear gradient-corrected correlation potential leads to results quite comparable to other sophisticated theoretical and experimental data available in the literature. In conclusion, the work-function approach coupled with the GPS numerical technique is shown to be a simple and efficient scheme for the accurate calculation of multiply excited atomic Rydberg states within DFT.

\section{ACKNOWLEDGMENTS}

This work was partially supported by the National Science Foundation under Contract No. PHY-0098106. We acknowledge the Kansas Center for Advanced Scientific Computing for the use of the Origin2400 supercomputer facilities sponsored by the NSF-MRI Program No. DMS-9977352.
[1] P. Hohenberg and W. Kohn, Phys. Rev. 136, B864 (1964).

[2] W. Kohn and L.J. Sham, Phys. Rev. 140, A1133 (1965).

[3] See, for example, R.G. Parr and W. Yang, Density-Functional Theory of Atoms and Molecules (Oxford University Press,
New York, 1989).

[4] Density Functional Theory, edited by E.K.U. Gross and R. M. Dreizler (Plenum, New York, 1995).

[5] A. Savin, C.J. Umrigar, and X. Gonze, Chem. Phys. Lett. 288, 
391 (1998).

[6] R. Singh and B.M. Deb, Phys. Rep. 311, 47 (1999).

[7] U. von Barth, Phys. Rev. A 20, 1693 (1979).

[8] T. Ziegler, A. Rauk, and E.J. Baerends, Theor. Chim. Acta 43, 261 (1977).

[9] T. Ziegler, Chem. Rev. 91, 651 (1991).

[10] C. Daul, Int. J. Quantum Chem. 52, 867 (1994).

[11] Á. Nagy, J. Phys. B 24, 4691 (1991); 29, 389 (1996).

[12] L. Fritsche, Phys. Rev. B 33, 3976 (1986).

[13] M. Petersilka, U.J. Gossmann, and E.K.U. Gross, Phys. Rev. Lett. 76, 1212 (1996).

[14] M. Petersilka and E.K.U. Gross, Int. J. Quantum Chem. 60, 181 (1996).

[15] M.E. Casida, C. Jamorski, K.C. Casida, and D.R. Salahub, J. Chem. Phys. 108, 4439 (1998).

[16] T. Grabo, M. Petersilka, and E.K.U. Gross, J. Mol. Struct.: THEOCHEM 501, 353 (2000).

[17] M.K. Harbola and V. Sahni, Phys. Rev. Lett. 62, 489 (1989).

[18] V. Sahni, Y. Li, and M.K. Harbola, Phys. Rev. A 45, 1434 (1992).

[19] A.K. Roy, R. Singh, and B.M. Deb, J. Phys. B 30, 4763 (1997).

[20] A.K. Roy, R. Singh, and B.M. Deb, Int. J. Quantum Chem. 65, 317 (1997).

[21] A.K. Roy and B.M. Deb, Phys. Lett. A 234, 465 (1997).

[22] A.K. Roy and B.M. Deb, Chem. Phys. Lett. 242, 461 (1998).

[23] R. Singh, A.K. Roy, and B.M. Deb, Chem. Phys. Lett. 296, 530 (1998).

[24] J.B. Krieger, Y. Li, and G.J. Iafrate, Phys. Rev. A 45, 101 (1992).

[25] G. Yao and S.I. Chu, Chem. Phys. Lett. 204, 381 (1993).

[26] J. Wang, S.I. Chu, and C. Laughlin, Phys. Rev. A 50, 3208 (1994).

[27] X. Chu and S.I. Chu, Phys. Rev. A 63, 013414 (2001).

[28] X.M. Tong and S.I. Chu, Chem. Phys. 217, 119 (1997).

[29] See, for example, X.M. Tong, and S.I. Chu, Phys. Rev. A 55, 3406 (1997).

[30] See, for example, X.M. Tong, and S.I. Chu, Phys. Rev. A 64, 013417 (2001).

[31] X. Chu and S.I. Chu, Phys. Rev. A 63, 023411 (2001).
[32] See, for example, X.M. Tong and S.I. Chu, Phys. Rev. A 61, 031401(R) (2000).

[33] M.E. Rose, Elementary Theory of Angular Momentum (Wiley, New York, 1957).

[34] C. Lee, W. Yang, and R.G. Parr, Phys. Rev. B 37, 785 (1988).

[35] E. Clementi and C. Roetti, At. Data Nucl. Data Tables 14, 179 (1974).

[36] A. Bürgers, D. Wintgen, and J.-M. Rost, J. Phys. B 28, 3163 (1995).

[37] C.F. Fischer, The Hartree-Fock Method for Atoms (Wiley, New York, 1977), p. 101.

[38] W.A. Goddard III, Phys. Rev. 176, 106 (1968).

[39] Z.-W. Wang, X.-W. Zhu, and K.T. Chung, Phys. Rev. A 46, 6914 (1992).

[40] J.S. Sims and S.A. Hagstrom, Phys. Rev. A 11, 418 (1975).

[41] A.W. Weiss, Phys. Rev. A 6, 1261 (1972).

[42] N. Koyama, H. Fukuda, T. Motoyama, and M.J. Matsuzawa, J. Phys. B 19, L331 (1986).

[43] E. Lindroth, Phys. Rev. A 49, 4473 (1994).

[44] Y.K. Ho, Phys. Rev. A 48, 3598 (1993).

[45] Y.K. Ho, J. Phys. B 12, L543 (1979).

[46] J.W. Cooper, U. Fano and F. Prats, Phys. Rev. Lett. 10, 518 (1963).

[47] A.D. Becke, Phys. Rev. A 38, 3098 (1998).

[48] V. Sahni, Phys. Rev. A 55, 1846 (1997).

[49] G.W.F. Drake, in Casimir Forces: Theory and Experiments on Atomic Systems, edited by F.S. Levin and D.A. Micha (Plenum, New York, 1993).

[50] G.W.F. Drake and Z.C. Yan, Chem. Phys. Lett. 229, 486 (1994).

[51] S. Bashkin and J.O. Stoner, Jr., Atomic Energy Levels and Grotrian Diagrams, Vol. 1 (North-Holland, Amsterdam, 1975); Addendum: Atomic Energy Levels and Grotrian Diagrams, Vol. 1 (North-Holland, Amsterdam, 1978).

[52] D. Begue, M. Merawa, and C. Pouchan, Phys. Rev. A 57, 2470 (1998).

[53] Y.K. Ho, Phys. Rev. A 23, 2137 (1981).

[54] D.R. Herrick and O. Sinanoglu, Phys. Rev. A 11, 97 (1975).

[55] H. Fukuda, N. Koyama, and M. Matsuzawa, J. Phys. B 20, 2959 (1987).

[56] P.J. Hicks and J. Comer, J. Phys. B 8, 1866 (1975). 